\title{
IMPROVED PHASE ESTIMATION BASED ON COMPLETE BISPECTRUM AND MODIFIED GROUP DELAY
}

\author{
${ }^{1}$ S.V. Narasimhan, ${ }^{1}$ Nandini Basu Mallick and ${ }^{2}$ Ratana Chaitanya \\ ${ }^{1}$ Aerospace Electronics and Systems Division, \\ National Aerospace Laboratory, Bangalore-560017, India \\ ${ }^{2}$ Dept. of Electronics and Communication Engineering, \\ National Institute of Technology, Karnataka Surathkal-57417, India
}

\begin{abstract}
In this paper, a new method for extracting the system phase from the bispectrum of the system output has been proposed. This is based on the complete bispectral data computed in the frequency domain and modified group delay. The frequency domain bispectrum computation improves the frequency resolution and the modified group delay reduces the variance preserving the frequency resolution. The use of full bispectral data also reduces the variance as it is used for averaging. For the proposed method at a signal to noise ratio of $5 \mathrm{~dB}$, the reduction in root mean square error is in the range of 1.5 to 7 times over the other methods considered.
\end{abstract}

\section{INTRODUCTION}

Bispectrum, the Fourier transform of the Triple Correlation Sequence (TCS) enables estimation of the deviation from the Gaussian behavior of a process, identification of a mixed phase system from its output and characterization of the nonlinear properties of the mechanism that generates the process [1, 3, 9]. These applications are due to the fact that the bispectrum provides information not only about the spectral magnitude but also about the complete spectral phase of the signal or system. On the other hand, the power spectrum has only information about the magnitude and provides that of the phase completely, only when the signal or system corresponds to a minimum phase. The complete phase information available in bispectrum is useful in applications like, recovery of reflection series in seismology [11], channel equalization in data communication [12], in nondestructive evaluation using ultrasonic methods [16] by deconvolving the defect impulse response from that of the measurement system in the presence of Gaussian noise due to characteristics of measuring instruments and propagation paths, and in signal or image reconstruction only from phase $[8,13]$.

In the bispectrum, though complete information is available, the system phase or wavelet phase has to be extracted from the bispectral phase and for this purpose many methods have been proposed [1,4,6-8]. Among these, a class of algorithms for phase recovery from partial (single slice) or complete bispectrum phase (all slices), which do not require phase unwrapping and solution of a system of equations, have been studied 
[7]. It has been observed that for high signal to noise ratio (SNR), their performance is similar. However at low SNR, the performance of the algorithms which use only the partial bispectrum is inferior to those based on complete bispectral phase information. In this direction, for phase estimation, use of many selected bispectral slices for averaging has been proposed [20]. This method though has the advantage of utilizing the a priori information for slice selection, since the averaging is done in the phase domain, it may result in the necessity of phase unwrapping and removal of linear phase component. Further, the selection of slices may result in instability. The present concern is about the method in which a relation between partial derivative of the bispectral phase and the system phase group delay (PGD) has been established and the system phase is obtained from the system group delay using the direct relation between them [6,7].

In spectral estimation, for achieving a good estimate of the spectrum, the group delay function has been modified [10]. In conventional periodogram spectral estimation, the data is segmented, windowed and their magnitude spectra are averaged and here the reduction in variance is at the cost of the frequency resolution due to the effect of window (segmentation). Basically, the variance of a spectral estimate is due to input noise driving a system, or associated noise with the signal, or data truncation effects. Any of these three or their combination, results in zeros close to the unit circle in the Z-plane and the fine structure or the variance in spectrum is due to these zeros. In the averaged windowed periodogram, the effect of these zeros is reduced as they are pulled towards the origin due to windowing. However, in addition to these zeros, the system or signal roots are also pulled inside and this leads to poor frequency resolution. But, the modified group delay removes the effect of the zeros which are close to the unit circle, without disturbing the system or signal roots and hence reduces the variance without compromising on frequency resolution. This desired feature of the modified group delay has been exploited for the phase estimation [17] using a single bispectral slice derived from the TCS. The modified group delay basically removes the truncation effects of the TCS (Gibbs ripple) without applying any common bispectral time domain window function. This reduces the Gibbs ripple preserving the frequency resolution of the rectangular window.

The use of partial bispectral data does not exploit full potential of the bispectrum in the estimation of the phase. The use of complete bispectral information enables averaging and hence effectively reduces the variance even when the signal to noise ratio is low. For the phase estimation based on group delay, full bispectral data has been used and improved performance even at low signal to noise ratio has been reported [2].

In all the above methods mentioned, the bispectrum has been computed from TCS and hence the maximum number of TCS lags used limits its frequency resolution. The windowing only further reduces the effective number of lags and hence the frequency resolution of the rectangular window. The modified group delay preserves only the frequency resolution corresponding to the number of considered TCS lags as no smoothing window is used for the TCS. Hence the computation of the bispectrum in the frequency domain which uses all possible lags for the given data length, significantly improves the frequency resolution or bias. Further, use of complete bispectral data and application of modified group delay very effectively reduce the variance even in the 
presence noise of Gaussian or any symmetric distribution, preserving the frequency resolution of a rectangular window used for the input signal.

In this paper, a new method for estimating the system phase from its output based on the modified group delay and the complete bispectrum data computed in the frequency domain has been proposed. The use of bispectral phase computed in frequency domain and the modified system group delay derived from it, not only improve the frequency resolution of the system phase estimate but also its variance. The frequency resolution is due to significantly more number of lags of the triple correlation being considered and also the removal of Gibbs ripple effect without using any smoothing window by the modified group delay. Further these reduce the variance as the modified group delay removes the zeros which are close to the unit circle which results in the undesired fine structure (variance) in the phase estimate and the full bispectral slices enable averaging over them. For the same reason, the proposed method has increased immunity to the associated noise (as noise manifests as increased variance). It has been found that in terms of root mean square error, the phase estimated by this approach is significantly superior even in the presence of noise to those obtained by the other methods based on the group delay derived from the bispectrum.

\section{GROUP DELAY FUNCTIONS}

\subsection{Original Group delay function:}

If $H(\omega)=|H(\omega)| e^{j \phi(\omega)}$ is a minimum phase system, then its magnitude and phase can be expressed as

$$
\begin{aligned}
& \ln |H(\omega)|=\sum_{n=0}^{\infty} c(n) \cos (\omega n) \\
& \text { and } \quad \phi(\omega)=-\sum_{n=0}^{\infty} c(n) \sin (\omega n)
\end{aligned}
$$

where $c(n)$ is the cepstral coefficient sequence and the magnitude and phase are related through this cepstral coefficient sequence. The group delay function, the negative derivative of the phase, is given by

$$
\tau_{p}(\omega)=\frac{-d \phi(\omega)}{d \omega}=\sum_{n=0}^{\infty} n c(n) \cos (\omega n)
$$

Given the system group delay, the system phase is uniquely computed from Eqn.(1b).

But if $H(\omega)$ is a mixed phase system, then the magnitude and phase cannot be expressed in terms of the same cepstral coefficients. 
Signal, Image and Video Processing, Online available: February 2008

$$
\begin{gathered}
\ln |H(\omega)|=\sum_{n=0}^{\infty} p(n) \cos (\omega n) \\
\phi(\omega)=-\sum_{n=0}^{\infty} q(n) \sin (\omega n)
\end{gathered}
$$

and

where $p(n)$ and $q(n)$ are cepstral coefficient sequences of the minimum phase equivalent signals derived from spectral magnitude and phase, respectively. The corresponding group delay functions are

$$
\begin{aligned}
& \tau_{m}(\omega)=\sum_{n=0}^{\infty} n p(n) \cos (\omega n) \\
& \tau_{p}(\omega)=\sum_{n=0}^{\infty} n q(n) \cos (\omega n)
\end{aligned}
$$

$\tau_{m}(\omega)$ and $\tau_{p}(\omega)$ are known as magnitude group delay (MGD) and the phase group delay functions, respectively.

From the system PGD $\tau_{p}(\omega)$, the phase is uniquely defined using Eqn.(2b) and there is no assumption of the values of the phase $\phi(\omega)$ at 0 and $\pi$ and there is no necessity of computing separately $\phi(\omega)$ at any particular value of $\omega$. For minimum phase signals, $p(n)=q(n)=c(n), \tau_{m}(\omega)=\tau_{p}(\omega)$; for maximum phase signals, $p(n)=-q(n), \tau_{m}(\omega)=-\tau_{p}(\omega)$ and for mixed phase signals, $p(n) \neq q(n)$, $\tau_{m}(\omega) \neq \tau_{p}(\omega)[8]$.

The significant values of MGD for a real pole are close to the origin and for a second order pole, are around the resonance frequency. The MGD for the zeros is same as those of poles but of opposite in sign. The MGD for a real and a complex pole is positive, but for a real and complex zero is negative. If $\left[\tau_{m}\left(e^{j \omega}\right)\right]^{+}$and $\left[\tau_{m}\left(e^{j \omega}\right)\right]^{-}$are the positive and negative parts of the MGD respectively, then the cepstral coefficients for the pole part, $p^{+}(n)$ and for the zero part, $p^{-}(n)$ are [15]

$$
\begin{gathered}
{\left[\tau_{m}\left(e^{j \omega}\right)\right]^{+}=C+\sum_{n=1}^{\infty} n p^{+}(n) \cos (\omega n)} \\
{\left[\tau_{m}\left(e^{j \omega}\right)\right]^{-}=C+\sum_{n=1}^{\infty} n p^{-}(n) \cos (\omega n)}
\end{gathered}
$$

$C$ is the average value that does not contribute to the shape of the spectrum. Cepstrally smooth spectra for poles and zeros can be obtained separately by considering only the first few cepstral coefficients in $p^{+}(n)$ and $p^{-}(n)$. 
Signal, Image and Video Processing, Online available: February 2008

\subsection{Modified magnitude group delay (MGDM)}

If $y(n)$ is a signal generated by an all-pole system, driven by a white noise and further, if its spectrum $Y(\omega)=N(\omega) / D(\omega)$, then $D(\omega)$ corresponds to the system or sinusoids and $N(\omega)$ to the excitation or the associated noise. For this case, the MGD is

$$
\tau_{m}(\omega)=\tau_{m N}(\omega)-\tau_{m D}(\omega)
$$

$\tau_{m N}(\omega)$ and $\tau_{m D}(\omega)$ are the MGDs for $N(\omega)$ and $D(\omega)$, respectively. Also, $\tau_{m}(\omega)$ is given by

$$
\tau_{m}(\omega)=\frac{Y_{m R}(\omega) X_{m R}(\omega)+Y_{m I}(\omega) X_{m I}(\omega)}{|Y(\omega)|^{2}}
$$

$Y_{m}(\omega)=F T\left[y_{m}(n)\right], X_{m}(\omega)=F T\left[x_{m}(n)\right], x_{m}(n)=n y_{m}(n) ; y_{m}(n)$ is the minimum phase equivalent of $y(n)$. Also,

$$
\tau_{m}(\omega)=\frac{\alpha_{N}(\omega)}{|N(\omega)|^{2}}-\frac{\alpha_{D}(\omega)}{|D(\omega)|^{2}}
$$

$\alpha_{N}(\omega)$ and $\alpha_{D}(\omega)$ are the numerator of the above Eqn. (5) for $\tau_{m N}(\omega)$, and $\tau_{m D}(\omega)$ respectively. The $\tau_{m N}(\omega)$ will have large amplitudes spikes due to very small values of $|N(\omega)|^{2}$ near the zeros which are close to unit circle and this is not so with $\tau_{m D}(\omega)$, as the roots of $D(\omega)$ are well within the unit circle. Hence, in $\tau_{m}(\omega)$, the effect of excitation or the associated noise masks the system or the signal component, which is assumed to be an all-pole one. The effect of these zeros could be reduced by multiplying $\tau_{m}(\omega)$ by $|N(\omega)|^{2}$. Also, as the envelope of $|N(\omega)|^{2}$ is nearly flat, the significant features of $\tau_{m D}(\omega)$ continue to exist, with the $|N(\omega)|^{2}$ fluctuations superimposed on it. Hence, the modified magnitude group delay (GDM) $\tau_{\text {mo }}(\omega)$ is,

$$
\tau_{m o}(\omega)=\tau_{m}(\omega)|N(\omega)|^{2}
$$

The estimate of $|N(\omega)|^{2}$ is given by $|\tilde{N}(\omega)|^{2}=|Y(\omega)|^{2} /|\bar{Y}(\omega)|^{2},|\bar{Y}(\omega)|^{2}$ is the cepstrally smoothed power spectrum obtained by considering only the initial few coefficients of the cepstral sequence.

\section{RELATION BETWEEN SYSTEM PHASE AND BISPECTRUM PHASE}

\subsection{The bispectrum}

The bispectrum of a process $y(k)$, generated by a system $H(v)$ driven by a zero mean non-Gaussian noise $w(k)$ with $E[w(k)]=\beta$, is given by Eqn.8. [1, 3, 5, 9] 
Signal, Image and Video Processing, Online available: February 2008

$$
B(\omega, v)=\sum_{m=-\infty}^{\infty} \sum_{n=-\infty}^{\infty} R(m, n) e^{-j(\omega m+v n)}
$$

$R(m, n)$ is the TCS and is given by

$$
R(m, n)=E[y(k) y(k+m) y(k+n)]
$$

Also,

$$
B(\omega, v)=B_{S}(\omega, v) B_{w}(\omega, v)
$$

$B_{s}(\omega, v)$ and $B_{w}(\omega, v)$ are the bispectra of the system $H(v)$ and of the input $w(k)$, respectively.

$$
B(\omega, v)=|B(\omega, v)| e^{j \psi(\omega, v)}
$$

Since $w(k)$ is a white noise sequence, its bispectral magnitude $\left|B_{w}(\omega, v)\right|=\beta$ and bispectral phase $\psi_{W}(\omega, v)=0$. Hence,

$$
\begin{gathered}
|B(\omega, v)|=\beta\left|B_{S}(\omega, v)\right| \\
\psi(\omega, v)=\psi_{S}(\omega, v)
\end{gathered}
$$

Since $\quad\left|B_{S}(\omega, v)\right|=|H(\omega)| \cdot|H(v)| \cdot|H(\omega+v)|$,

$$
|B(\omega, v)|=\beta \cdot|H(\omega)| \cdot|H(v)| \cdot|H(\omega+v)|
$$

For $\omega=0$, Eqn.(12a) becomes

$$
\begin{gathered}
|H(v)|^{2}=\frac{B(0, v)}{\beta|H(0)|}, \quad|H(0)| \neq 0 \\
\psi_{s}(\omega, v)=\phi(\omega)+\phi(v)-\phi(\omega+v)
\end{gathered}
$$

$\phi(v)$ is the phase of the system $H(v)$ and $H(v)=|H(v)| e^{j \phi(v)}$

\subsection{For a single bispectral phase slice data}

The system bispectral phase $\psi_{s}(\omega, v)$ can be obtained from a single slice of $\psi(\omega, v)$, the bispectral phase of the output of the system [4]. The partial derivative of $\psi(\omega, v)$ with respect to ' $\omega$ ' is given by

$$
\frac{\partial \psi(\omega, v)}{\partial \omega}=\frac{\partial \phi(\omega)}{\partial \omega}-\frac{\partial \phi(\omega+v)}{\partial \omega}
$$


Signal, Image and Video Processing, Online available: February 2008

at $\omega=0$,

$$
\left.\frac{\partial \psi(\omega, v)}{\partial \omega}\right|_{\omega=0}=\left.\frac{\partial \phi(\omega)}{\partial \omega}\right|_{\omega=0}-\left.\frac{\partial \phi(\omega+v)}{\partial v}\right|_{\omega=0}
$$

since, $\left.\quad \frac{\partial \phi(\omega+v)}{\partial \omega}\right|_{\omega=0}=\left.\frac{\partial \phi(\omega+v)}{\partial v}\right|_{\omega=0}$

For any given phase function $\theta(\xi)$, the PGD $\tau_{p}(\xi)$ is the negative derivative of phase with respect to ' $\xi$ ' and is given by

$$
\tau_{p}(\xi)=\frac{-d \theta(\xi)}{d \xi}
$$

Hence, Eqn (14) can be written as $\tau_{p \omega}(0, v)=\tau_{p}(0)-\tau_{p}(v)$

$$
\text { or, } \tau_{p}(v)=-\left[\tau_{p \omega}(0, v)-\tau_{p}(0)\right] \quad, \quad \text { where, } \tau_{p \omega}(\omega, v)=\frac{-\partial \psi(\omega, v)}{\partial \omega}
$$

$\tau_{p}(0)$ is the value of $\tau_{p}(\omega)$ at $\omega=0$ and hence it is constant with respect to " $v$ ". Given $\tau_{p \omega}(0, v)$, the system group delay $\tau_{p}(v)$ can be obtained by subtracting $\tau_{p}(0)$ from $\tau_{p \omega}(0, v)$ and further changing the sign. The mean value of $\tau_{p \omega}(0, \omega)$ corresponds to the linear phase component in the phase and it has to be removed to get the desired phase. Once the PGD of the system is estimated, the system phase is uniquely defined and no assumptions regarding the values of $\phi(v)$ at $v=0$ and $\pi$ are required.

\subsection{By using complete bispectral phase:}

Holambe has modified the method of deriving the system group delay from single slice of bispectral phase for utilizing the full bispectral phase data [2]. This is advantageous since it provides an accurate estimate of the system group delay by reducing its variance and also with better immunity to the associated noise if any.

The partial derivative of $\psi(\omega, v)$ with respect to ' $\omega$ ' is given by (Eqn.(13)),

$$
\frac{\partial \psi(\omega, v)}{\partial \omega}=\frac{\partial \phi(\omega)}{\partial \omega}-\frac{\partial \phi(\omega+v)}{\partial \omega}
$$

Integrating on both sides with respect to $v$

$$
\int_{-\pi}^{\pi} \frac{\partial \psi(\omega, v)}{\partial \omega} \partial v=\int_{-\pi}^{\pi} \frac{\partial \phi(\omega)}{\partial \omega} \partial v-\int_{-\pi}^{\pi} \frac{\partial \phi(\omega+v)}{\partial \omega} \partial v
$$

Since the first integral on the right hand side, is independent of $v$, 
Signal, Image and Video Processing, Online available: February 2008

$$
\begin{gathered}
\int_{-\pi}^{\pi} \frac{\partial \psi(\omega, v)}{\partial \omega} \partial v=2 \pi\left[\frac{\partial \phi(\omega)}{\partial \omega}\right]-\int_{-\pi}^{\pi} \frac{\partial \phi(\omega+v)}{\partial \omega} \partial v \\
\tau_{p \omega}(\omega)=\frac{1}{2 \pi} \int_{-\pi}^{\pi} \tau_{p \omega}(\omega, v) d v+\frac{1}{2 \pi} \int_{-\pi}^{\pi} \tau_{p \omega}(\omega+v) d v
\end{gathered}
$$

In $\tau_{p \omega}(\omega), \tau_{p \omega}(\omega+v)$ and $\tau_{p \omega}(\omega, v)$; the differentiation is with respect to $\omega$. The linear phase does not contribute to the bispectral phase, since for the linear phase corresponding to a delay $t_{0}$,

$$
\psi_{s}(\omega, v)=\phi(\omega)+\phi(v)-\phi(\omega+v)=-\omega t_{0}-v t_{0}-\left\{-(\omega+v) t_{0}\right\}=0
$$

Since the system phase $\phi(\omega)$ does not contain any linear phase and $\tau_{p}(\omega)$ is periodic for all values of $\omega$, the second term in the R.H.S of Eqn. (15b) which represents the average value of the group delay is zero. Therefore

$$
\begin{array}{r}
\tau_{p \omega}(\omega)=\frac{1}{2 \pi} \int_{-\pi}^{\pi} \tau_{p \omega}(\omega, v) d v \\
\text { or }, \quad \frac{\partial \phi(\omega)}{\partial \omega}=\frac{1}{2 \pi} \int_{-\pi}^{\pi} \frac{\partial \psi(\omega, v)}{\partial \omega} \partial v
\end{array}
$$

The discrete version of the above relation (dropping $\omega$ in the subscript for simplicity) is

$$
\tau_{p}(\omega)=-\frac{1}{L} \sum_{v=0}^{L-1} T(\omega, v)
$$

$T(\omega, v)=\frac{\partial \psi(\omega, v)}{\partial \omega}, L:$ Number of slices which is same as the DFT size.

\section{IMPROVED PHASE ESTIMATION BY BISPECTRUM AND MODIFIED GROUP DELAY}

In the phase estimation by GD approach, the frequency resolution and hence the variance are decided by the type of the window applied to the TCS and the number of data segments considered in the ensemble. This is so since the window reduces the effective lag length and sharpness of truncation (reduction in sidelobe level for non rectangular window). However here, the reduction in variance is at the cost of frequency resolution for a given lag length.

To improve the frequency resolution it is necessary to consider as many lags as possible for a given data segment length. This motivates the bispectrum to be computed in the frequency domain where all the possible lags are directly available. However, this results in an increased variance. This is due to the fact that the variances for higher lags 
are relatively more than those for lower lags. In order to preserve the frequency resolution and also to reduce the variance of the bispectrum derived in the frequency domain, the modified group delay approach is an appropriate one. In the approaches which use TCS, the variance is reduced due to reduction in frequency resolution resulting from limited number of TCS lags. But still the estimate will suffer from variance due to abrupt truncation, the Gibbs ripple effect. The use of any smooth window function in an attempt to reduce the variance due to Gibbs ripple will further reduce the frequency resolution, for a given length of data. In the new approach, the total variance effect both due to higher frequency resolution and the Gibbs ripple (due to data segmentation) is taken care of by the modified group delay. Further, the use of full bispectral data instead of a single slice, improves not only the variance reduction but also the robustness of the estimation to the associated noise.

For a good estimation of the bispectrum, among many bispectral windows compared to the rectangular window, specifically the optimal window reduces the number of segments to be required for averaging. Such a bispectral estimate has good frequency resolution and reduced variance, as the window is derived by minimizing the mean square error. The optimal window has the best possible smoothness to reduce the variance and the best possible flatness to provide improved frequency resolution [14].

For applying the modification for the GD, the estimated system PGD from the partial derivative of the complete bispectral phase is treated as MGD. The equivalent magnitude spectrum derived from this MGD corresponds to the positive part of the PGD, i.e., to the poles and maximum phase zeros of the system. However, the modification for the GD effectively removes the negative part of the PGD. That is, the information about the minimum phase zeros is lost. To get an improved estimate of the GD corresponding to minimum phase zeros, the PGD is inverted (the sign of the PGD is changed) and the modification for the GD is applied. From this, the negative part of the PGD is obtained. The improved complete PGD estimate is obtained by subtracting the improved PGD for the minimum phase zeros from the improved PGD for the poles and maximum phase zeros. Any discontinuity in the estimate of the PGD can be smoothed by considering only the first few cepstral coefficients of the improved complete PGD. From this improved complete PGD, the phase is determined as they are directly related.

It is of the opinion that the magnitude estimate will be of better accuracy than the phase as even small errors in it may not be tolerable. In view of this, the phase correction can be made to the above PGD derived, using the MGD. For this, the regions of MGD corresponding to PGD are identified and the MGD values with suitable sign modifications are replaced in the PGD. This is explained in detail below.

Let $\left[\tau_{m}(\omega)\right]^{+}$and $\left[\tau_{m}(\omega)\right]^{-}$represent the positive and negative parts of $\tau_{m}(\omega)$, respectively. Also let $\left[\tau_{p}(\omega)\right]^{+}$and $\left[\tau_{p}(\omega)\right]^{-}$, be the positive and negative parts of $\tau_{p}(\omega)$, respectively. The errors in estimating the phase by nonparametric approach affect the location of both poles and zeros. For a physically realizable system, all the poles must 
be of minimum phase and the poles correspond to the positive portion of the MGD. The MGD will contain complete information about the poles. In the PGD, the correction for the poles $[18,19]$ is done as

$$
\left[\tau_{p}(\omega)\right]^{+}=\left[\tau_{m}(\omega)\right]^{+} \text {, if } \tau_{m}(\omega)>0
$$

However phase correction for zeros requires the information about their locations with respect to unit circle whether they are inside (minimum phase) or outside (maximum phase). The PGD provides this information, as the PGD is positive for a maximum phase zero and negative for a minimum phase zero. For a minimum phase zero, the phase correction is achieved by

$$
\left[\tau_{p}(\omega)\right]^{-}=\left[\tau_{m}(\omega)\right]^{-} \text {if } \tau_{m}(\omega)<0 \text { and } \tau_{p}(\omega)<0
$$

For a maximum phase zero, the positive part of PGD (other than that for poles) is replaced with the corresponding negative part of MGD with change of sign.

$$
\begin{gathered}
\text { Let } \Gamma(\omega)=\left[\tau_{p}(\omega)\right]^{+}-\left[\tau_{m}(\omega)\right]^{+} \text {then, } \\
{\left[\tau_{p}(\omega)\right]^{+}=-\left[\tau_{m}(\omega)\right]^{-} \text {if } \tau_{m}(\omega)<0 \text { and } \Gamma(\omega)>0 \text {, }}
\end{gathered}
$$

Hence the corrected PGD is,

$$
\tau_{p c}(\omega)=\left[\tau_{p}(\omega)\right]^{+}+\left[\tau_{p}(\omega)\right]^{-}
$$

For the corrected PGD, $\tau_{p c}(\omega), q_{1}(k)=\operatorname{IDFT}\left[\tau_{p c}(\omega)\right]$,

$$
q(k)=\left\{\begin{array}{lc}
q_{1}(k), & k=0, L / 2 \\
2 q_{1}(k) / k, & k=1,2, \cdots L / 2-1 \\
0, & k=L / 2+1, \ldots L-1
\end{array}\right.
$$

$q(k)$ : Cepstral coefficients derived from the corrected PGD. $L$ is the DFT length. These corrections may result in some discontinuity in the corrected $\operatorname{PGD} \tau_{p c}(\omega)$ and can be reduced by considering only first few Fourier coefficients $M(M<<L)$ of the PGD and the smoothed corrected PGD $\tilde{\tau}_{p c}\left(e^{j \omega}\right)$ is

$$
\tilde{\tau}_{p c}\left(e^{j \omega}\right)=\sum_{k=1}^{M} k q(k) \cos (\omega k)
$$

Then, the system phase is

$$
\hat{\phi}\left(e^{j \omega}\right)=-\sum_{k=1}^{M} q(k) \sin (\omega k)
$$


The number of cepstral coefficients used should be sufficient to get only the gross feature of the spectrum as the cepstral coefficients are the Fourier coefficients of the log spectrum. As their number increases, the FT of the truncated cepstrum will be closer to the original spectrum (i.e., which includes all the cepstral coefficients). If the normalized error energy $E_{n}(m)$ considering only the first $q$ cepstral coefficients $c(k)$ is,

$$
E_{n}(m)=\sum_{k=m+1}^{\infty} c^{2}(k) / \sum_{k=1}^{\infty} c^{2}(k)
$$

then, initially $E_{n}(m)$ will decrease sharply as $m$ is increased but after a certain value of $m=M$, the knee point, the decrease is not appreciable. This implies that number of cepstral coefficients even lower than $M$ provides a good representation of the spectrum.

In the proposed method, the modifications to the group delay are applied in the system group delay domain and not in bispectrum domain. Since the modified group delay removes the zeros close to unit circle due to associated or driving white noise, it has built in noise immunity and there is no necessity to identify the noisy regions in the bispectrum like in some of the existing methods [20].

\subsection{Computational complexity}

Based on the algorithm for FFD-GDM method given in the appendix, the computational requirement in terms of FFTs is examined.

i) For the bispectrum computation using $N_{S}$ segments, $N_{S}$ FFTs of length $L$ are required.

ii) For computing the phase group delay (including phase correction) 3 FFTs and 2 IFFTs are required.

iii) To modify the system group delay obtained from the bispectrum full data, 8 FFTs and 6 IIFTs are required. Further to get system phase from the system group delay 1 FFT and 1 IFFT are necessary.

Totally it requires $N_{S}$ plus 12 FFTs and 9 IFFTs for the algorithm.

For single slice using frequency domain computation of the bispectrum, same computational load is required. It is important to note that for using full data the only extra operation required is averaging and this takes $(L-1)$ additions and one division. In this respect, for the improvement in the performance achieved by using full data, the extra computational load involved is very meager. 
Signal, Image and Video Processing, Online available: February 2008

\section{SIMULATION RESULTS}

The performance of the phase extraction by the proposed method is illustrated for different systems and is compared to those of other methods considered. For convenience of presentation, the different methods considered are labeled as below:

Bispectrum single slice derived from TCS using optimal window [4] : TSS-OW Bispectrum single slice derived from TCS and GDM [17] : TSS-GDM Bispectrum single slice derived in frequency domain with GDM $\quad$ : FSS-GDM Bispectral full data derived from TCS and GDM Bispectral full data derived in frequency domain and GDM

: TFD-GDM

: FFD-GDM.

Each system is driven by a zero-mean white noise having one-sided exponential distribution. Further to see the effect of associated noise on the performance, Gaussian noise has been added to the output of the systems. The performance measures, namely, the sample mean $(E[\phi(v)])$ and the root mean square error (RMSE) $\sigma$, computed for $K$ ensembles are given by:

$$
E[\phi(v)]=\frac{1}{K} \sum_{i=1}^{K} \phi_{i}(v) \quad \text { and } \quad \sigma=\left\{\frac{1}{K} \sum_{i=1}^{K}\left[\phi_{i}(v)-\phi(v)\right]^{2}\right\}^{1 / 2}
$$

where, $\phi(v)$ and $\phi_{i}(v)$ are the ideal phase (can be computed from system transfer function as its parameters are assumed to be known) and its estimate at $i^{t h}$ trial. $K$ is the number of trials used in computing the mean and RMSE and in this study, value of $K=50$ is used for all examples considered. Further to quantify the performance, the normalized sum of the sample mean square error (NSSMSE), expressed as a percentage, is also used as a performance index. It is given by:

$$
\text { NSSMSE }=\frac{\sum_{v} \sigma(v)^{2}}{\sum_{v} \phi(v)^{2}} \times 100
$$

The NSSMSE values for phase estimation of each system by different methods are listed in Table-1. The various examples that have been considered are:

Example-1: $\quad H_{1}(z)=\frac{\left(1-0.4 z^{-1}\right)}{(1-0.64 z)\left(1+0.6 z^{-1}\right)}$

The system $H_{1}(z)$ is an ARMA system with mixed phase poles. For this system, the bispectrum is estimated by using number of data segments $N_{s}=40$, segment length $L_{s}$ $=128$ samples and the partial derivative of the bispectral phase is estimated using a Discrete Fourier Transform (DFT) of length $\mathrm{L}=128$ samples. $\mathrm{N}_{\mathrm{s}}$ and $\mathrm{L}_{s} / \mathrm{L}$ decide the variance and frequency resolution, respectively. The number of lags used for computing the TCS is $\mathrm{M}=\mathrm{N}=10$, as these provide the necessary frequency resolution and keep the variance at low level. 
Example-2: $\quad H_{2}(z)=\frac{(1-0.64 z)\left(1-0.6 z^{-1}\right)}{\left(1+0.7 z^{-1}\right)\left(1+0.6 z^{-1}\right)}$

The system $H_{2}(z)$ is an ARMA system with mixed phase zeros. For this system, $\mathrm{N}_{\mathrm{s}}=40, \mathrm{~L}_{\mathrm{s}}=128, \mathrm{~L}=128$ and $\mathrm{M}=\mathrm{N}=12$.

Example-3: $\quad H_{3}(z)=\frac{(1-0.86 z)\left(1-0.84 z^{-1}\right)}{\left(1+0.96 z^{-1}\right)\left(1+0.8 z^{-1}\right)}$

The system $H_{3}(z)$ is an ARMA system having mixed phase zeros, with roots close to the unit circle. For this system, $\mathrm{N}_{\mathrm{s}}=40, \mathrm{~L}_{\mathrm{s}}=256, \mathrm{~L}=256$ and $\mathrm{M}=\mathrm{N}=11$. In this case, a larger DFT length is required to resolve the phase curve as the system has roots close to unit circle.

Example-4: $\quad H_{4}(z)=\frac{1}{\left(1-2.7377 z^{-1}+3.7476 z^{-2}-2.6293 z^{-3}+0.9224 z^{-4}\right)}$

The system $H_{4}(z)$ is an AR system with minimum phase complex poles. For this system, $\mathrm{N}_{\mathrm{s}}=48, \mathrm{~L}_{\mathrm{s}}=256, \mathrm{~L}=256$ and $\mathrm{M}=\mathrm{N}=13$. Since, the system has complex poles, larger values of $N_{s}$ and $L_{s} / L$ are required to reduce the variance and to resolve the phase curve.

Table-1: NSSMSE performance comparison for different methods

\begin{tabular}{|c|c|c|c|c|c|c|}
\hline Example & CPM & TSS-OW & TSS-GDM & TFD-GDM & FSS-GDM & FFD-GDM \\
\hline$H_{1}(z)$ & - & 1.9424 & 1.1837 & 0.5220 & 0.5562 & 0.2536 \\
\hline $\begin{array}{c}H_{1}(z) \\
(\mathrm{SNR} 5 \mathrm{~dB})\end{array}$ & - & 2.8256 & 1.6347 & 0.7927 & 0.9124 & 0.4205 \\
\hline$H_{2}(z)$ & - & 3.9248 & 3.0008 & 1.6891 & 2.0516 & 1.2066 \\
\hline $\begin{array}{c}H_{2}(z) \\
(\mathrm{SNR} 5 \mathrm{~dB})\end{array}$ & - & 5.0000 & 3.7124 & 1.9836 & 2.6162 & 1.4331 \\
\hline$H_{3}(z)$ & - & 7.9086 & - & 5.2737 & - & 4.2343 \\
\hline $\begin{array}{c}H_{3}(z) \\
(\mathrm{SNR} 4 \mathrm{~dB}) \\
\end{array}$ & - & 11.0257 & - & 8.5170 & - & 7.5016 \\
\hline$H_{4}(z)$ & 1.9358 & - & - & 13.5799 & - & 5.1207 \\
\hline $\begin{array}{c}H_{4}(z) \\
(\mathrm{SNR} 10 \mathrm{~dB})\end{array}$ & 62.9206 & - & - & 15.0485 & - & 8.8382 \\
\hline
\end{tabular}



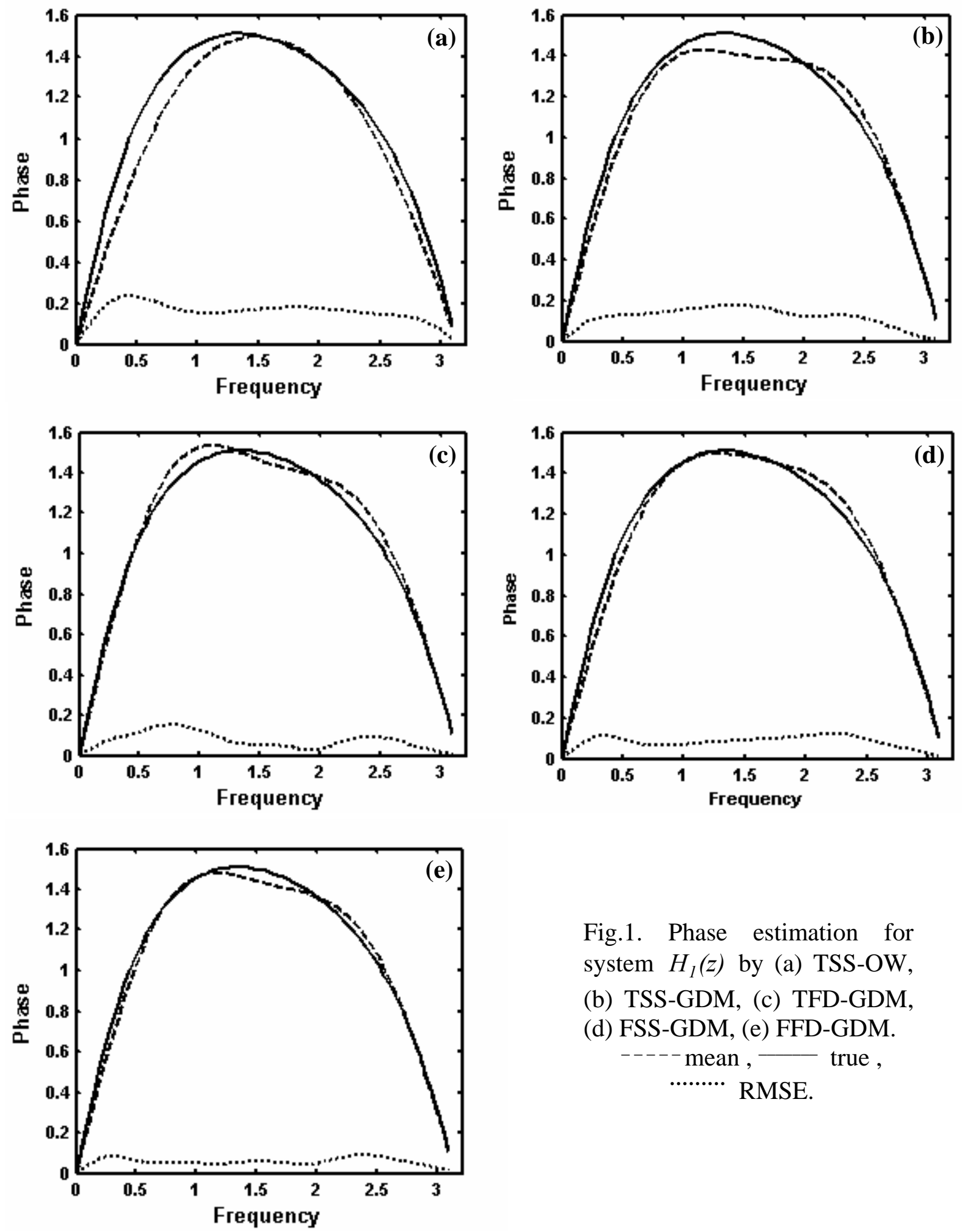

Fig.1. Phase estimation for system $H_{1}(z)$ by (a) TSS-OW, (b) TSS-GDM, (c) TFD-GDM, (d) FSS-GDM, (e) FFD-GDM. ----- mean, - true, RMSE. 

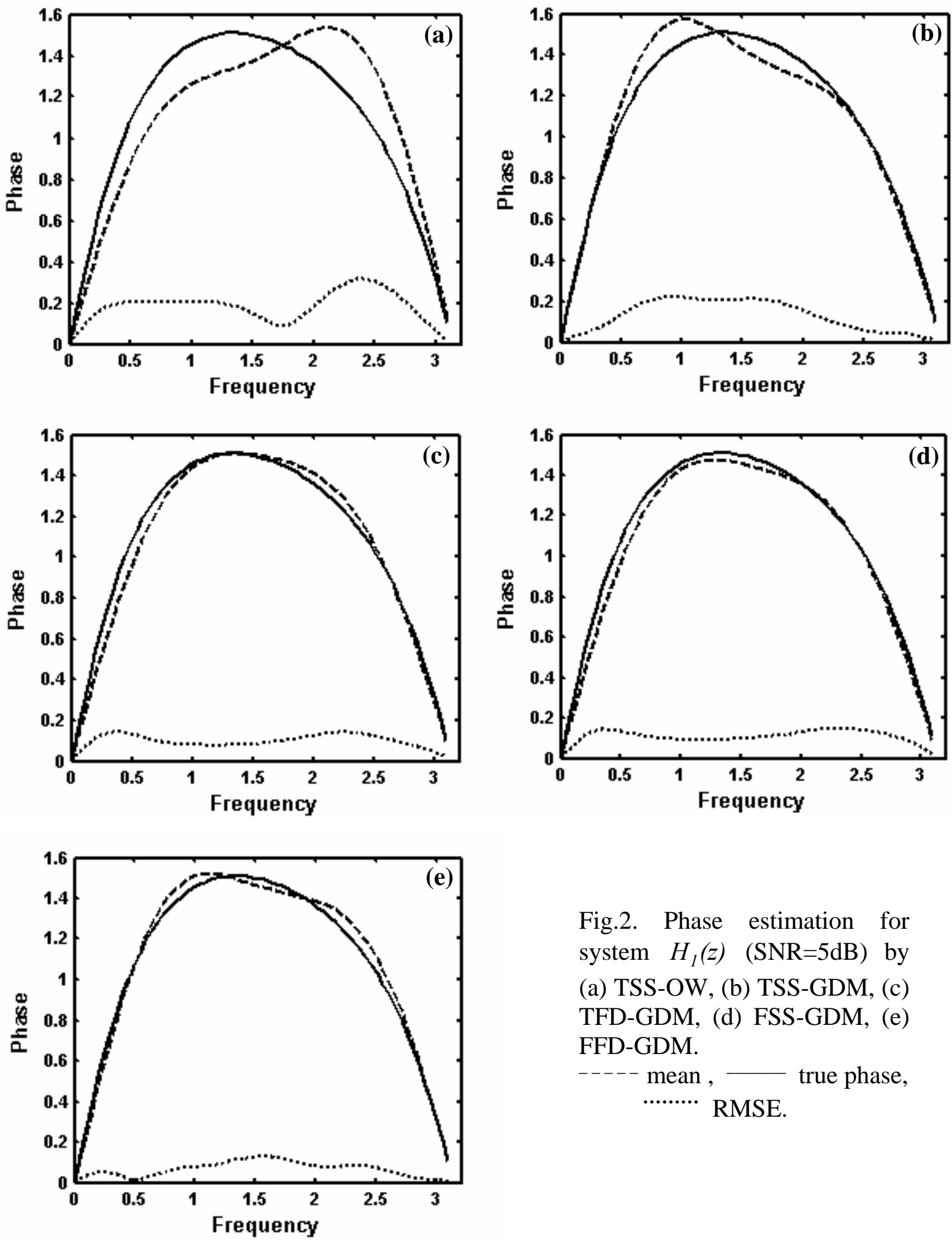

Fig.2. Phase estimation for system $H_{1}(z) \quad(\mathrm{SNR}=5 \mathrm{~dB})$ by (a) TSS-OW, (b) TSS-GDM, (c) TFD-GDM, (d) FSS-GDM, (e) FFD-GDM.

----- mean , - true phase,

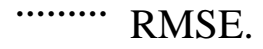


Signal, Image and Video Processing, Online available: February 2008
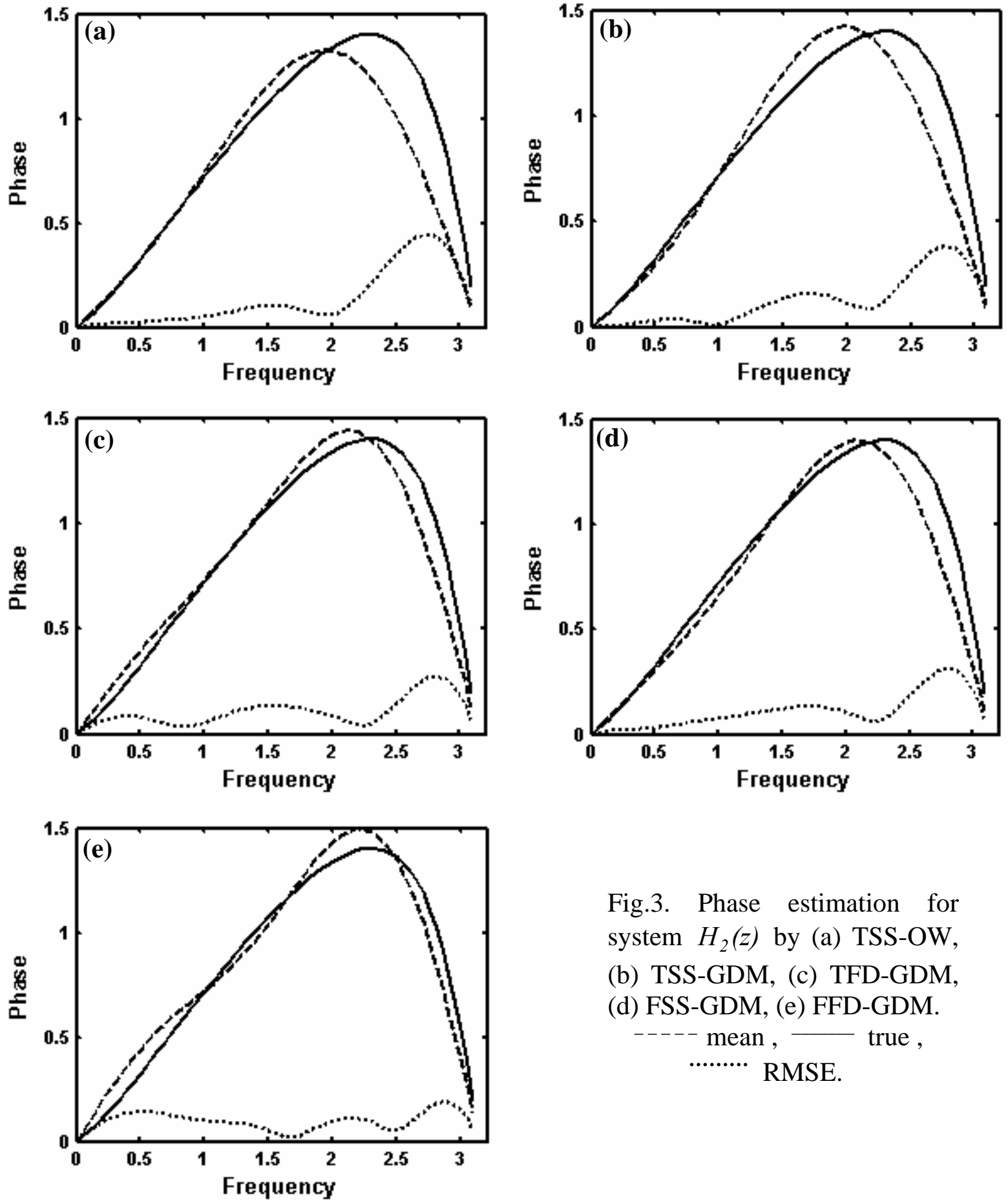

Fig.3. Phase estimation for system $\mathrm{H}_{2}(z)$ by (a) TSS-OW, (b) TSS-GDM, (c) TFD-GDM, (d) FSS-GDM, (e) FFD-GDM. ---- mean, - true, RMSE. 
Signal, Image and Video Processing, Online available: February 2008
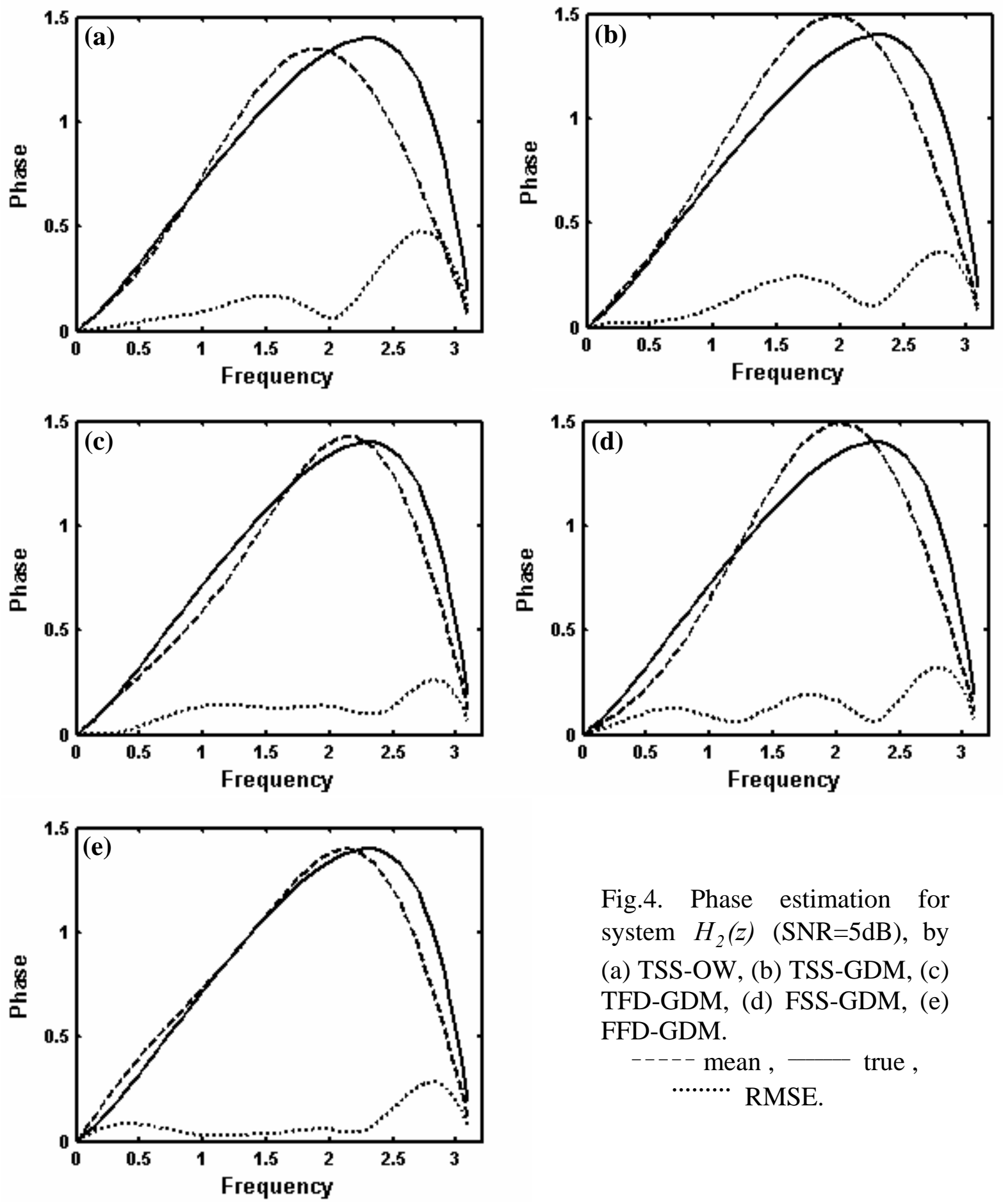

Fig.4. Phase estimation for system $\mathrm{H}_{2}(z) \quad(\mathrm{SNR}=5 \mathrm{~dB})$, by (a) TSS-OW, (b) TSS-GDM, (c) TFD-GDM, (d) FSS-GDM, (e) FFD-GDM.

$$
\begin{aligned}
& \text {........ } \\
& \text { RMSE. }
\end{aligned}
$$



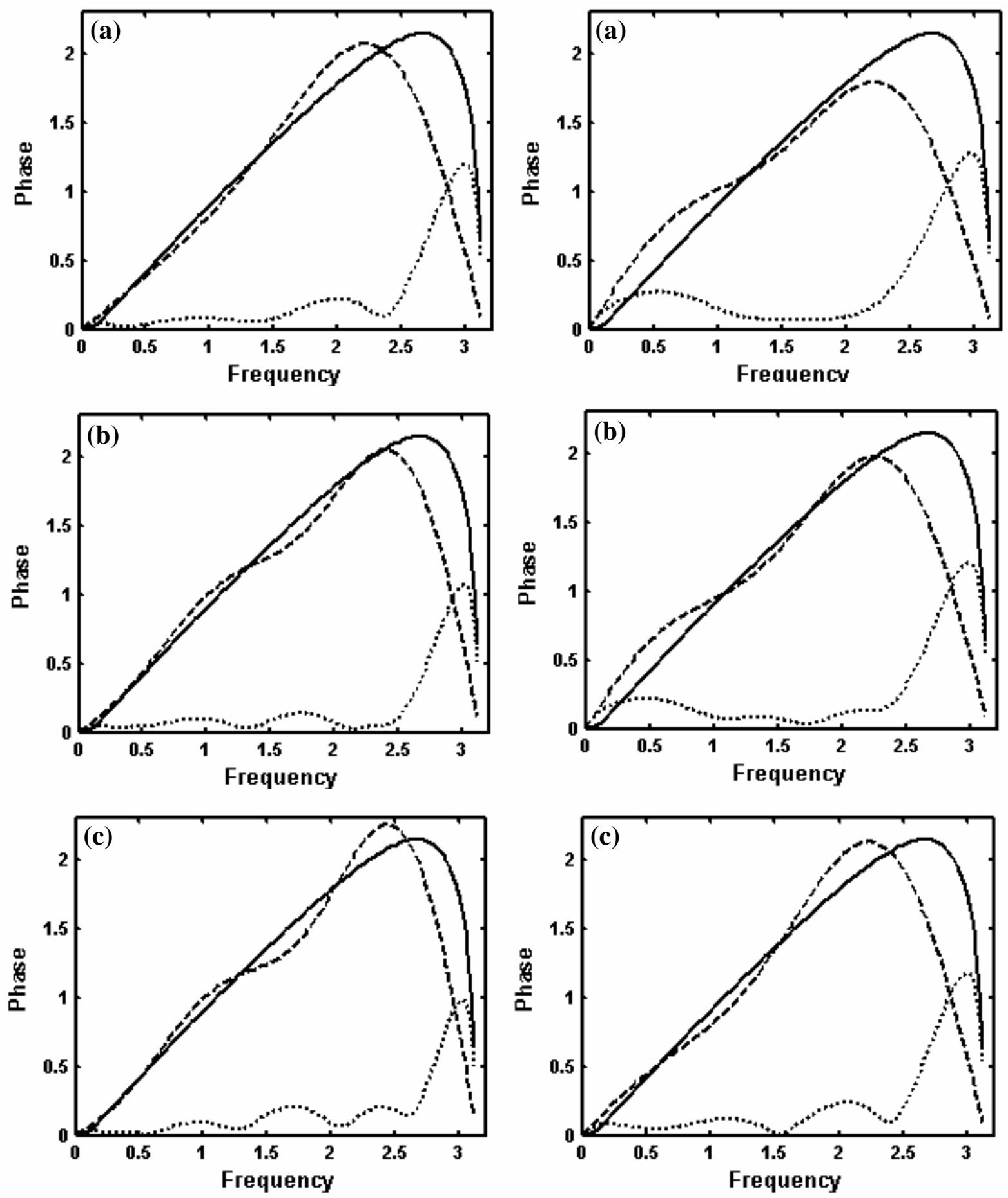

Fig.5. Phase estimation for system $\mathrm{H}_{3}(z)$ by (a) TSS-OW, (b) TFD-GDM, (c) FFD-GDM. ----- mean, - true , RMSE.

Fig.6. Phase estimation for system $H_{3}(z) \quad(\mathrm{SNR}=4 \mathrm{~dB})$ by (a) TSS-OW, (b) TFD-GDM, (c) FFD-GDM. 

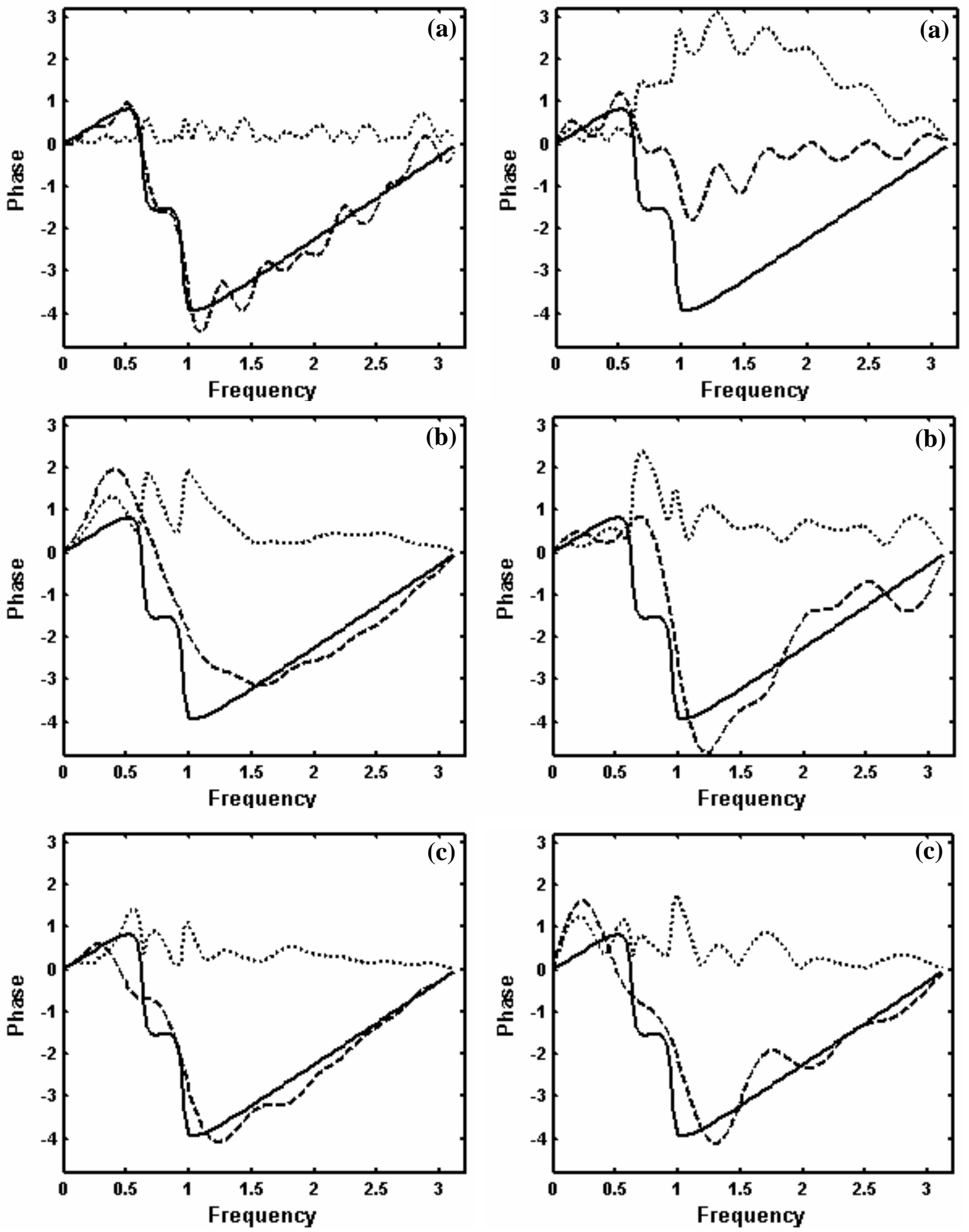

Fig.7. Phase estimation for system $H_{4}(z)$ by (a) CPM, (b) TFD-GDM, (c) FFD-GDM.

$$
\begin{aligned}
& -\cdots \cdot \text { mean , } \\
& \text {........ } \\
& \text { RMSE. }
\end{aligned}
$$

Fig.8. Phase estimation for system $H_{4}(z)(\mathrm{SNR}=10 \mathrm{~dB})$ by (a) CPM, (b) TFD-GDM, (c) FFD-GDM.

$$
---- \text { mean , - true, }
$$

….... RMSE. 
Signal, Image and Video Processing, Online available: February 2008

The mean and RMSE of the phase estimates of systems $H_{1}(z)$ and $H_{2}(z)$ using different methods, are shown in Figs. 1 and 3 respectively. The mean plot obtained by TSS-OW deviates from the ideal phase curve more than that by TSS-GDM and TFD-GDM (Figs $1(a, b, c)$ and $3(a, b, c))$. This is due to the fact that better frequency resolution is achieved when modified group delay is used to reduce the variance, instead of the optimal window. In the case of FSS-GDM and FFD-GDM, there is significant match between the mean and the true phase as compared to the time domain methods (Figs 1(d, e) and 3(d, e)), owing to the increased number of lags offered by the computation of the bispectrum in the frequency domain. The RMSE for TSS-OW and for TSS-GDM are of comparable magnitude (Figs 1(a, b) and 3(a, b)). However, the RMSE by TFD-GDM is lower than those by TSS-OW and TSS-GDM (Figs 1(c) and 3(c)) because of the additional variance reduction obtained by use of full bispectrum data. For the same reason, the RMSE values by FFD-GDM are lower than that by FSS-GDM (Figs 1(d, e) and 3(d, e)). For the system $H_{1}(z)$, the NSSMSE by the proposed method is only 0.13 times of that by TSSOW. For system $\mathrm{H}_{2}(z)$, the NSSMSE by the proposed method is about 0.3 times that by TSS-OW is (Table-1).

Figs.2 and 4 represent the mean and RMSE of the phase estimates of systems $H_{1}(z)$ and $H_{2}(z)$ when their outputs are corrupted by Gaussian noise. The performance of phase estimation methods using bispectrum single slice deteriorates in the presence of noise, leading to high RMSE values (Figs. 2(a, b, d) and 4(a, b, d)). The estimation methods that use full bispectral data are less sensitive to noise than those based on single bispectrum slice, yielding lower error (Figs. 2(c, e) and 4(c, e)). For an SNR of 5dB, the NSSMSE of the phase estimate of system $H_{1}(z)$ by TSS-OW is about 7 times more than that by the proposed method. The improvement factor by the proposed method over TSSOW, for system $\mathrm{H}_{2}(z)$ with SNR=5dB is 3.5 (Table-1).

For the system $H_{3}(z)$, which has roots close to unit circle (Example-3), phase estimation by FFD-GDM has been compared with those by TSS-OW and TFD-GDM. The mean phase by FFD-GDM (Fig.5(c)) matches the ideal phase to a greater extent than that by TSS-OW (Fig.5(a)) and TFD-GDM (Fig.5(b)). The NSSMSE of the phase estimate by FFD-GDM is $1 / 2$ of that by TSS-OW. In presence of noise, the performance of FFD-GDM (Fig.6(c)) and TFD-GDM (Fig.6(b)) is better than that of TSS-OW (Fig.6(a)). The improvement factor by the proposed method over TSS-OW for an SNR of $4 \mathrm{~dB}$ is 1.5 (Table-1), which is less compared to those for Examples 1 and 2 because of the effect of zeros close to the unit circle.

The proposed method has been compared with several other bispectrum based methods in the previous examples. Since the system $H_{4}(z)$ is a minimum phase system, its phase can be estimated by the conventional periodogram method (CPM) also. Hence phase estimation by the bispectrum based methods TFD-GDM (Fig 7(b)) and FFD-GDM (Fig 7(c)) are compared with that obtained by CPM (Fig 7(a)). For this system, the performance of TFD-GDM is much poorer than CPM. This can be attributed to the 
Signal, Image and Video Processing, Online available: February 2008

reduced frequency resolution of TFD-GDM due to limited number of lags which is not so with the CPM. This is also indicated by the mean plot as it is unable to resolve the two peaks (the absence of kink) (Fig.7 (a, b)). The FFD-GDM has a better performance than that of TFD-GDM both in terms of RMSE and mean. But its performance is inferior to that of CPM (Fig.7 (a, c)) and this may be due to additional averaging involved, which increases the bias. In presence of noise with an SNR of 10dB, the proposed method performs better than CPM by a factor of 7 ! Also, it is almost 1.7 times better than TFDGDM (Table-1). Although the conventional periodogram method is efficient for phase estimation of minimum phase systems in the absence of noise; its performance significantly deteriorates in the presence of noise (Fig.8(a)). Phase estimation methods using the bispectrum provide better noise rejection as compared to the conventional method (Fig.8(b,c)). Among the bispectrum-based methods, the proposed method yields the best results.

These results substantiate the superior performance of the proposed method, even in the presence of noise and with the system roots close to the unit circle (Example-3). This method is also significantly better than other methods for phase estimation of minimum phase systems in presence of noise (Example-4).

\section{CONCLUSIONS}

A new method for extracting the system phase from the bispectrum of the system output, based on bispectrum and modified group delay, was proposed. In this method, the bispectrum was computed in the frequency domain and full bispectral data was used. Computing bispectrum in frequency domain improves frequency resolution, but with increased variance. The modified magnitude group delay, due to removal of zeros close to the unit circle, and the use of full bispectral data, as it facilitates averaging, significantly contribute to the reduction in variance, without affecting the frequency resolution. For the same reason, they significantly improve the immunity of the estimate to noise. Thus it doesn't require any specific considerations for noisy regions in the bispectrum. It has been observed that the proposed method yields significantly better results than by the other methods, even for low SNRs and for roots close to the unit circle, as the factor of improvement is in the range of 1.5 to 7 . Also, for the phase estimation of minimum phase systems in presence of noise, it is 7 times better than the conventional periodogram method.

\section{APPENDIX}

The algorithm for the proposed method is given as:

1. Compute average bispectrum, $B(\omega, v)$, from available data.

(i) Segment the data $y(n)$ into $N_{s}$ segments of $L_{s}$ samples each.

$$
\text { Let, } y_{j}^{(l)}=y\left(j+(l-1) L_{s}\right)
$$


Signal, Image and Video Processing, Online available: February 2008

where $l=1 \ldots \ldots . . N_{s}, j=1, \ldots \ldots \ldots . . L_{s}$

(ii) Generate

$$
B(\omega, v)=\frac{1}{N_{s}} \sum_{l=1}^{N s} Y^{l}(\omega) Y^{l}(v) Y^{l}(\omega+v)
$$

where $Y^{l}(\omega)$ is the DFT of $y^{l}(n)$.

2. Compute the bispectral phase $\psi\left(l_{1}, l_{2}\right)$.

3. From the bispectral phase, compute $T(l, u)$ the negative bispectral phase derivative (Bispectral PGD) with respect to $l[8]$.

(i) Generate the all-pass sequence $g_{1}\left(k_{1}, k_{2}\right)$

$$
g_{1}\left(k_{1}, k_{2}\right)=\operatorname{IDFT}\left[e^{j \psi\left(l_{1}, l_{2}\right)}\right]
$$

(ii) Generate $g_{2}\left(k_{1}, k_{2}\right)$, where $g_{2}(0,0)=0$,

$$
\begin{gathered}
g_{2}\left(k_{1}, k_{2}\right)=k_{1} g_{1}\left(k_{1}, k_{2}\right), \text { for } k_{1}=0,1, \ldots \ldots \frac{L}{2} \text { and for } k_{2}=0,1, \ldots . . L-1 \\
g_{2}\left(k_{1}, k_{2}\right)=\left(k_{1}-L\right) g_{1}\left(k_{1}, k_{2}\right), \text { for } k_{1}=L / 2+1, \ldots \ldots L-1 \text { and for } k_{2}=0,1, \ldots . L-1
\end{gathered}
$$

(iii) $T(l, u)=\operatorname{real}\left[G_{1}(l, u)\right] \operatorname{real}\left[G_{2}(l, u)\right]+\operatorname{imag}\left[G_{1}(l, u)\right] \operatorname{imag}\left[G_{2}(l, u)\right]$, where $G_{1}\left(l_{1}, l_{2}\right)$ and $G_{2}\left(l_{1}, l_{2}\right)$ are the DFTs of $g_{1}\left(k_{1}, k_{2}\right)$ and $g_{2}\left(k_{1}, k_{2}\right)$, respectively.

4. Compute the PGD, $\tau_{p}(l)$.

$$
\tau_{p}(l)=-\frac{1}{L} \sum_{u=0}^{L-1} T(l, u)
$$

5. Compute the corrected PGD from MGD.

(i) Calculate the magnitude estimate $H(l)$ using Eqn.(12b).

$$
|H(l)|^{2}=\frac{B(0, l)}{\beta|H(0)|}
$$

(ii) Compute the MGD, $\tau_{m}(l)$ using Eqns. (2a) and (3).

(iii) Phase correction for poles:

$$
\left[\tau_{p}(\omega)\right]^{+}=\left[\tau_{m}(\omega)\right]^{+} \text {, if } \tau_{m}(\omega)>0 .
$$

(iv) Phase correction for minimum phase zeros:

$$
\left[\tau_{p}(\omega)\right]^{-}=\left[\tau_{m}(\omega)\right]^{-} \text {if } \tau_{m}(\omega)<0 \text { and } \tau_{p}(\omega)<0 \text {. }
$$


Signal, Image and Video Processing, Online available: February 2008

(v) Phase correction for maximum phase zeros:

$$
\begin{aligned}
& \left.\qquad \tau_{p}(\omega)\right]^{+}=-\left[\tau_{m}(\omega)\right]^{-} \text {if } \tau_{m}(\omega)<0 \text { and } \Gamma(\omega)>0, \\
& \text { where, } \quad \Gamma(\omega)=\left[\tau_{p}(\omega)\right]^{+}-\left[\tau_{m}(\omega)\right]^{+} .
\end{aligned}
$$

(vi) Compute the corrected PGD as,

$$
\tau_{p c}(\omega)=\left[\tau_{p}(\omega)\right]^{+}+\left[\tau_{p}(\omega)\right]^{-}
$$

6. Compute the equivalent magnitude $A_{u m}(\omega)$ from the corrected PGD.

$$
\begin{gathered}
q_{1}(k)=\operatorname{IDFT}\left[\tau_{p c}(l)\right] \\
A_{u m}(\omega)=\sum_{n=0}^{L-1} q(k) \cos (\omega n)
\end{gathered}
$$

with

$$
q(k)=\left\{\begin{array}{lc}
q_{1}(k), & k=0, L / 2 \\
2 q_{1}(k) / k, & k=1,2, \cdots L / 2-1 \\
0, & k=L / 2+1, \ldots L-1
\end{array}\right.
$$

7. Compute smoothed version of $A_{u m}(\omega), A_{s m}(\omega)$ by considering only the first few cepstral coefficients.

8. Compute $A_{d m}=A_{u m}-A_{s m}$.

9. Get the modified group delay (GDM) $\tau_{m m}(\omega)$ by multiplying PGD with $\exp \left(2 A_{d m}(\omega)\right)$.

10. Compute the equivalent magnitude, $A_{m m}$ from $\tau_{m m}(\omega)$.

11. Compute the scaled equivalent magnitude.

$$
\begin{aligned}
& \text { A } A_{m s}=\frac{A_{m m}}{G}+\operatorname{mean}\left(A_{u m}\right) \\
& \text { where, } \quad G=\frac{\max \left[A_{m m}-\operatorname{mean}\left(A_{m m}\right)\right]}{\max \left[A_{u m}-\operatorname{mean}\left(A_{u m}\right)\right]}
\end{aligned}
$$

12. Compute GD corresponding to poles and maximum phase zeros $\tau_{p p}(\omega)$ from this scaled equivalent magnitude. 
Signal, Image and Video Processing, Online available: February 2008

13. Similarly, compute GD corresponding to minimum phase zeros $\tau_{p n}(\omega)$ from the scaled equivalent magnitude obtained by reversing original PGD using steps 6 to 11 .

14. Compute $\tau_{p m}(\omega)=\tau_{p p}(\omega)-\tau_{p n}(\omega)$.

15. Compute phase $\phi(\omega)$ from $\tau_{p m}(\omega)$ using Eqns. (2b) and (4).

\section{REFERENCES}

[1] M.R. Raghuveer and C.L. Nikias, "Bispectrum estimation: A digital signal processing framework”, Proceedings of IEEE, Vol. 75, No.7, pp.869-89, 1987.

[2] R.S. Holambe, A.K. Ray, and T.K. Basu, "Signal phase recovery using the bispectrum”, Signal Processing, 55, pp.321-337, Aug.1996.

[3] C.L. Nikias and Athina P. Petropulu, "Higher order spectral analysis: A non-linear signal processing framework”, Prentice-Hall, Signal Processing Series, 1993.

[4] S.V. Narasimhan, G.R. Reddy, E.I. Plotkin and M.N.S. Swamy, "Phase estimation by bispectrum: A group delay approach”, Signal Processing, Vol.27, No.1, pp.7986, 1992.

[5] M.R. Raghuveer and C.L. Nikias, "Bispectrum estimation: A parametric approach", IEEE Trans. Acoustics Speech and Signal Processing, Vol.33, No.4, pp.1213-1229, Oct.1985.

[6] R. Pan and C.L. Nikias, "The Complex cepstrum of higher order cumulants and non-minimum phase system identification”, IEEE Trans. on Acoustics Speech and Signal Processing, Vol.36, No.2, pp.186-204, feb.1988.

[7] S.V. Narasimhan, G.R. Reddy, E.I. Plotkin, and M.N.S. Swamy, "Bispectrum based mixed phase system identification by AR/ARMA models: AR/ARMA models: A group delay approach”, IEEE Trans. Circuits and Systems, Vol.39, No.9, pp.671674, Sept.1992.

[8] B. Yegnanarayana, D.K. Saikia, and T.R. Krishnan, "Significance of group delay functions in signal reconstruction from spectral magnitude or phase", IEEE Trans. ASSP, Vol.32, No. 3, pp.610-623, June.1984.

[9] C.L. Nikias and J.M. Mendel, "Signal processing with higher order spectra”, IEEE Signal Processing Magazine, pp. 10-37, July 1993. 
Signal, Image and Video Processing, Online available: February 2008

[10] B. Yegnanarayana and Hema A. Murthy, "Significance of group delay functions in spectrum estimation”, IEEE Trans. Signal Processing, Vol. 40, No. 9, pp.22812289, Sept.1992.

[11] T. Matsuoka and T. J. Ulrych, "Phase estimation using the bispectrum", Proc. IEEE, Vol. 72, pp.1403-1411, 1984.

[12] Benveniste, M. Goursat and G. Ruget, "Robust identification of non-minimum phase system: Blind adjustment of linear equalizer in data communications”, IEE Trans. Automat. Control, Vol. AC-25, pp. 385-398, June 1980.

[13] A.V. Oppenheim, M.H. Hayes and J.S. Lim, "Iterative procedures for signal reconstruction from Fourier Transform phase”, Optical Engineering, Vol. 21, pp. 122-127, February 1982.

[14] T. Subba Rao and M.M. Gabbar, "An introduction to bispectral analysis and bilinear time series models”, in: D.R. Brillinger et al. eds., Lecture Notes on Statistics 24, Springer, Berlin, 1984.

[15] Yegnanarayana, "Speech analysis by pole-zero decomposition of short time spectra”, Signal Processing, Vol.17, pp. 141-150, 1989.

[16] Yamani, "Flaw impulse response estimation in ultrasonic non-destructive evolution using bi-cepstra”, NDT\&E International, Vol. 40, pp. 57-61, 2007.

[17] M. Hazarathaiah and S.V. Narasimhan, "Phase estimation based on bispectrum and modified group delay", Signal Processing and Communication (SPCOM 2001), Bangalore, Organized by the IEEE Signal Processing Society, Bangalore Chapter.

[18] S.V. Narasimhan and P.V.S. Giridhar, "Improved system blind identification based on second order cyclostatinary statistics: A group delay approach", Academy Proceedings in Engineering Sciences, SADHANA, 25(112), Vol. 25, Part 2, pp. 8596, April, 2000.

[19] S.V. Narasimhan, M. Hazarathaiah and P.V.S. Giridhar, "Channel blind identification based on cyclostationarity and group delay", Signal Processing, Vol.85, No. 7, pp. 1275-1286, July 2005.

[20] Athina P. Petropulu and Udantha R. Abeyratne, "System reconstruction from higher order spectra slices”, IEEE Transactions on Signal Processing, Vol. 45, No. 9, September 1997. 